\title{
A graph-theoretic approach to sparse matrix inversion for implicit differential algebraic equations
}

\author{
H. Yoshimura \\ Department of Applied Mechanics and Aerospace Engineering, Waseda University, Ohkubo, Shinjuku, \\ Tokyo 169-8555, Japan \\ Correspondence to: H. Yoshimura (yoshimura@waseda.jp)
}

Received: 15 November 2012 - Revised: 9 March 2013 - Accepted: 5 April 2013 - Published: 6 June 2013

\begin{abstract}
In this paper, we propose an efficient numerical scheme to compute sparse matrix inversions for Implicit Differential Algebraic Equations of large-scale nonlinear mechanical systems. We first formulate mechanical systems with constraints by Dirac structures and associated Lagrangian systems. Second, we show how to allocate input-output relations to the variables in kinematical and dynamical relations appearing in DAEs by introducing an oriented bipartite graph. Then, we also show that the matrix inversion of Jacobian matrix associated to the kinematical and dynamical relations can be carried out by using the input-output relations and we explain solvability of the sparse Jacobian matrix inversion by using the bipartite graph. Finally, we propose an efficient symbolic generation algorithm to compute the sparse matrix inversion of the Jacobian matrix, and we demonstrate the validity in numerical efficiency by an example of the stanford manipulator.
\end{abstract}

\section{Introduction}

Multibody systems such as space structures, manipulators, etc. are known to be represented as implicit mechanical systems with kinematical constraints, holonomic or nonholonomic, which may be eventually expressed by implicit nonlinear Differential-Algebraic Equations (DAEs). In particular, for the numerical integration of such DAEs, we need to employ stiffly stable implicit numerical integrators such as Backward Differentiation Formula (see, Hachtel et al., 1971; Brayton et al., 1972), since the DAEs are to be highly nonlinear and stiff in general. On the other hand, one may face at a serious problem in CPU time for solving the implicit nonlinear algebraic equations, especially, for the case of large-scale systems. Namely, increasing degrees of freedom of the system, it eventually requires much CPU time in computing the matrix inversion of Jacobian matrix of the implicit DAEs in Newton's iteration at each time, since the Jacobian matrix of discretized nonlinear algebraic equations may be random sparse in general.

A major stumbling block lies in the fact that the Jacobian matrix has the random sparseness as well as highly nonlinear in terms of generalized coordinates. So far, some nu- merical technique of sparse matrix inversions for VLSI circuits or networks has been developed by using the blocktriangularization of matrices (see, for instance, Orlandea et al., 1977a; Murata et al., 1985), where a structural analysis is effectively made by means of graph and matroid theory (for instance, refer to Murota, 2000). In these conventional approaches, one may properly find out pivots in the Gaussian elimination process at each time step in an ad hoc way (where we note that the choice of pivots is quite relevant with input-output relations as will be shown shortly). This eventually requires much CPU time to calculate the inversion of the Jacobian matrix unless utilizing some effective sparse matrix algorithms. Namely, it is almost impossible to figure out a prior fill-in and fill-out in Gaussian elimination since topological structure of such a sparse Jacobian matrix might be so much random and complicated. Thus, we need to develop an efficient numerical algorithm of sparse matrix inversion for solving large-scale implicit DAEs in a systematic way.

In this paper, we develop a graph-theoretic approach to computing sparse matrix inversion for large-scale nonlinear mechanical systems by using Dirac structures and associated implicit Lagrangian systems. The underlying idea is to regard a mechanical system as an interconnected system of 
elements, in which systems are comprised of constitutive relations of physical elements, structural relations among the physical relations, and causal (input-output) relations among physical variables. In particular, focusing upon the inputoutput relations associated with all the kinematical and dynamical relations of original mechanical systems, we develop bipartite graphs and then we show how the sparse matrix inversion can be made by effectively using the input-output relations. Furthermore, we explain solvability for the sparse Jacobian matrix inversion associated to the DAEs by using the bipartite graph. Finally, we propose an efficient and systematic symbolic generation algorithm to compute the sparse matrix inversion of the Jacobian matrix and we demonstrate its validity in numerical efficiency by an example of the stanford manipulator.

\section{Implicit Lagrangian systems}

Let us review Dirac structures and associated implicit Lagrangian systems by following Yoshimura and Marsden (2006a,b, 2008).

\subsection{Dirac structures}

Let $Q$ be an $n$-dimensional configuration manifold, whose kinematical constraints are given by a constraint distribution $\Delta_{Q} \subset T Q$, given by, at each $q \in Q$,

$\Delta_{Q}(q)=\left\{v \in T_{q} Q \mid\left\langle\omega^{a}(q), v\right\rangle=0, a=1, \ldots, m\right\}$,

where $\omega^{a}$ are $m$ one-forms on $Q$. Define the distribution $\Delta_{T^{*} Q}$ on $T^{*} Q$ by

$\Delta_{T^{*} Q}=\left(T \pi_{Q}\right)^{-1}\left(\Delta_{Q}\right) \subset T T^{*} Q$,

where $T \pi_{Q}: T T^{*} Q \rightarrow T Q$ is the tangent map of the cotangent bundle projection $\pi_{Q}: T^{*} Q \rightarrow Q$, while the annihilator of $\Delta_{T^{*} Q}$ can be defined by, for each $z \in T_{q}^{*} Q$,

$$
\begin{aligned}
\Delta_{T^{*} Q}^{\circ}(z)=\left\{\alpha_{z} \in T_{z}^{*} T^{*} Q \mid\left\langle\alpha_{z},\right.\right. & \left.w_{z}\right\rangle=0 \\
& \text { for all } \left.w_{z} \in \Delta_{T^{*} Q}(z)\right\} .
\end{aligned}
$$

Let $\Omega$ be the canonical symplectic structure on $T^{*} Q$ and $\Omega^{b}$ : $T T^{*} Q \rightarrow T^{*} T^{*} Q$ be the associated bundle map. Then, a Dirac structure $D_{\Delta_{Q}}$ on $T^{*} Q$ induced from $\Delta_{Q}$ can be defined by, for each $z \in T_{q}^{*} Q$,

$$
\begin{aligned}
D_{\Delta_{Q}}(z) & =\left\{\left(w_{z}, \alpha_{z}\right) \in T_{z} T^{*} Q \times T_{z}^{*} T^{*} Q \mid\right. \\
& \left.w_{z} \in \Delta_{T^{*} Q}(z) \text { and } \alpha_{z}-\Omega^{\mathrm{b}}(z) \cdot w_{z} \in \Delta_{T^{*} Q}^{\circ}(z)\right\} .
\end{aligned}
$$

\subsection{Local representations}

Let us choose local coordinates $q^{i}$ on $Q$ so that $Q$ is locally represented by an open set $W \subset \mathbb{R}^{n}$. The constraint set $\Delta_{Q}$ defines a subspace of $T Q$, which we denote by $\Delta(q) \subset \mathbb{R}^{n}$ at each point $q \in W$. If the dimension of $\Delta(q)$ is $n-m$, then we can choose a basis $e_{m+1}(q), e_{m+2}(q), \ldots, e_{n}(q)$ of $\Delta(q)$.

Recall that the constraint sets can be also represented by the annihilator of $\Delta(q)$, which is denoted by $\Delta^{\circ}(q)$ spanned by such one-forms that we write as $\omega^{1}, \omega^{2}, \ldots, \omega^{m}$. Using $\pi_{Q}: T^{*} Q \rightarrow Q$ locally denoted by $z=(q, p) \mapsto q$ and $T \pi_{Q}$ : $T T^{*} Q \rightarrow T Q ;(q, p, \dot{q}, \dot{p}) \mapsto(q, \dot{q})$, it follows that

$\Delta_{T^{*} Q} \cong\{(q, p, \dot{q}, \dot{p}) \mid q \in U, \dot{q} \in \Delta(q)\}$.

Let points in $T^{*} T^{*} Q$ be locally denoted by $(q, p, \beta, u)$, where $\beta$ is a covector and $u$ is a vector. Then, the annihilator of $\Delta_{T^{*} Q}$ is locally represented as

$\Delta_{T^{*} Q}^{\circ} \cong\left\{(q, p, \beta, u) \mid q \in U, \beta \in \Delta^{\circ}(q)\right.$ and $\left.u=0\right\}$.

Since we have the local formula $\Omega^{b}(q, p) \cdot w_{(q, p)}=(q, p,-\dot{p}, \dot{q})$, the condition $\alpha_{(q, p)}-\Omega^{b}(q, p) \cdot w_{(q, p)} \in \Delta_{T^{*} Q}^{\circ}$ reads $\alpha+\dot{p} \in$ $\Delta^{\circ}(q)$, and $w-\dot{q}=0$, where $\alpha_{(q, p)}=(q, p, \alpha, w)$ and $w_{(q, p)}=$ $(q, p, \dot{q}, \dot{p})$. Thus, the induced Dirac structure is locally represented by

$$
\begin{aligned}
D_{\Delta_{Q}}(q, p)=\{((\dot{q}, \dot{p}),(\alpha, w)) \mid \dot{q} \in \Delta(q), & \\
w & \left.=\dot{q}, \alpha+\dot{p} \in \Delta^{\circ}(q)\right\} .
\end{aligned}
$$

Representation (I): let us introduce a matrix representation of $D_{\Delta_{Q}}$ given in Eq. (2). First, let $N^{T}(q)$ be an $n \times m$ matrix whose $m$-column vectors $\omega^{1}(q), \ldots, \omega^{m}(q)$ span the basis of $\Delta^{\circ}(q)$, namely, $N^{T}(q)=\left[\omega^{1}(q), \ldots, \omega^{m}(q)\right]$ and the distribution $\Delta(q) \subset \mathbb{R}^{n} \cong T_{q} Q$ may be represented by

$\Delta(q)=\left\{\dot{q} \in \mathbb{R}^{n} \mid N(q) \dot{q}=0\right\}$.

Using Lagrange multipliers $\lambda=\left(\lambda_{1}, \ldots, \lambda_{m}\right) \in \mathbb{R}^{m}$, one has

$\Delta^{\circ}(q)=\left\{\beta \in\left(\mathbb{R}^{n}\right)^{*} \mid \beta=N^{T}(q) \lambda\right\}$.

Thus, the induced Dirac structure can be represented by

$$
\begin{array}{r}
D_{\Delta_{Q}}(q, p)=\{((\dot{q}, \dot{p}),(\alpha, w)) \mid N(q) \dot{q}=0, \\
\left.w=\dot{q}, \alpha+\dot{p}=N^{T}(q) \lambda\right\} .
\end{array}
$$

Representation (II): as shown in Eq. (3), for Representation (I) for the induced Dirac structure, we utilized the Lagrange multipliers, which represent constraint forces in constrained mechanical systems. Here, we develop another representation of $D_{\Delta_{Q}}$ on $T^{*} Q$ without using the Lagrange multipliers.

Let us choose an $n \times(n-m)$ matrix $B(q)=$ $\left[e_{m+1}(q), \ldots, e_{n}(q)\right]$, whose column vectors span the basis of $\Delta(q)$. Then, it follows that the distribution $\Delta(q) \subset \mathbb{R}^{n} \cong T_{q} Q$ can be also represented by

$\Delta(q)=\left\{\dot{q} \in \mathbb{R}^{n} \mid \dot{q}=B(q) u\right\}$,

where $u=\left(u^{m+1}, \ldots, u^{n}\right) \in \mathbb{R}^{n-m}$. Note that the orthogonality condition between $N(q)$ and $B(q)$ holds:

$B^{T}(q) N^{T}(q)=0$. 
The above condition naturally comes from the fact that $\Delta^{\circ}$ is the annihilator of the distribution $\Delta$; namely, in other words, the basis $e_{m+1}(q), \ldots, e_{n}(q)$ is orthogonal to the dual basis $\omega^{1}(q), \ldots, \omega^{m}(q)$ at each $q \in Q$. Therefore, one can read that

$\Delta^{\circ}(q)=\left\{\beta \in\left(\mathbb{R}^{n}\right)^{*} \mid B^{T}(q) \beta=0\right\}$.

Thus, the induced Dirac structure $D_{\Delta_{Q}} \subset T T^{*} Q \oplus T^{*} T^{*} Q$ can be represented without using the Lagrange multipliers as

$$
\begin{array}{r}
D_{\Delta_{Q}}(q, p)=\{((\dot{q}, \dot{p}),(\alpha, w)) \mid N(q) \dot{q}=0, \\
\left.w=\dot{q}, B^{T}(q)(\alpha+\dot{p})=0\right\} .
\end{array}
$$

\subsection{Ehresmann connection and structural relations}

We briefly review an Ehresmann connection associated with nonholonomic mechanical systems; as to the details, for example, refer to Yoshimura and Marsden (2006b).

Assume that there is a bundle structure with a projection $\pi: Q \rightarrow \mathcal{R}$ for $Q$; that is, there exists another manifold $\mathcal{R}$ called the base. We call the kernel of $T_{q} \pi$ at any point $q \in Q$ the vertical space denoted by $\mathcal{V}_{q}$. An Ehresmann connection $A$ is a vertical vector-valued one-form on $Q$, which satisfies

1. $A_{q}: T_{q} Q \rightarrow \mathcal{V}_{q}$ is a linear map at each point $p \in Q$,

2. $A$ is a projection : $A\left(v_{q}\right)=v_{q}$, for all $v_{q} \in \mathcal{V}_{q}$.

Thus, we can split the tangent space at $q$ such that $T_{q} Q=$ $\mathcal{H}_{q} \oplus \mathcal{V}_{q}$, where $\mathcal{H}_{q}=\operatorname{Ker} A_{q}$ is the horizontal space at $q$.

Suppose there exist nonholonomic constraints $\Delta_{Q} \subset T Q$, which are given by $m(<n)$ algebraic equations for $n$ generalized velocity vector $v=\dot{q}=\left(\dot{q}^{1}, \ldots, \dot{q}^{n}\right) \in \Delta(q) \subset T_{q} Q$ as in Eq. (1). Let us choose an Ehresmann connection $A$ such that $\mathcal{H}_{q}=\Delta_{Q}(q)$ or we assume that the connection is chosen such that the constraints are written as $A \cdot v_{q}=0$, where the constraint distribution $\Delta_{Q}$ is spanned by a set of $m$ independent one-forms, which is given, in local coordinates $q^{i}=\left(r^{\alpha}, s^{a}\right)$ for $Q$, by

$\omega^{a}=\mathrm{d} s^{a}-J_{\alpha}^{a}(r, s) \mathrm{d} r^{\alpha}$.

In a matrix representation,

$N(q) v=\left[I_{m} \mid-\mathcal{J}(q)\right]\left[\frac{v^{\circ}}{v^{*}}\right]=o_{m}$,

where $v$ is locally split into dependent velocity $v^{\circ}=\dot{q}^{\circ}=$ $\left(\dot{q}^{1}, \ldots, \dot{q}^{m}\right)$ and $v^{*}=\dot{q}^{*}=\left(\dot{q}^{m+1}, \ldots, \dot{q}^{n}\right)$ independent velocity and $\mathcal{J}$ is a submatrix associated with the constraints. Geometrically speaking, this splitting corresponds to a choice of Ehresmann connections for the given constraints (see Yoshimura and Marsden, 2006b).

Corresponding to the annihilator, one has the dynamical relations associated to the generalized force vector $Q=$ $\left(Q_{1}, \ldots, Q_{n}\right) \in \Delta^{\circ}(q) \subset T_{q}^{*} Q$ dual to $v$ as

$B^{T}(q) Q=\left[\mathcal{J}(q)^{T} \mid I_{n-m}\right]\left[\frac{Q^{\circ}}{Q^{*}}\right]=o_{n-m}$,

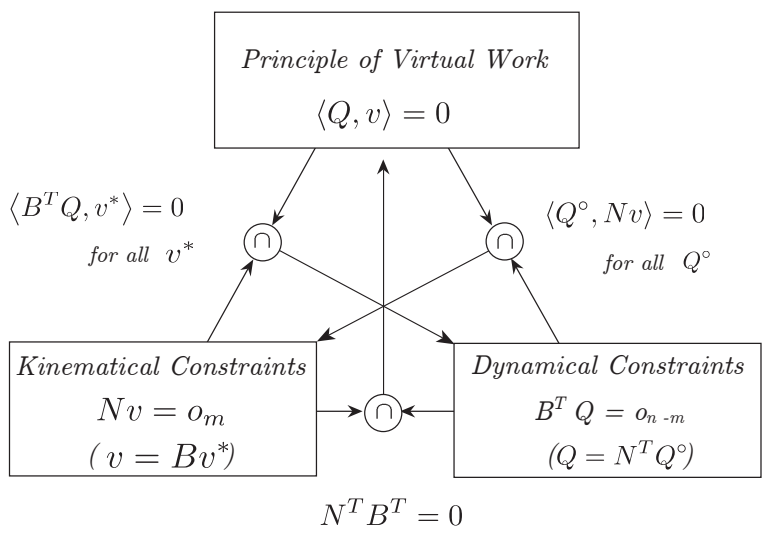

Figure 1. Duality principle.

where $\Delta_{Q}^{\circ}$ denotes the annihilator of $\Delta_{Q}$, and $Q^{\circ}=\left(Q_{1}, \ldots, Q_{m}\right)$ and $Q^{*}=\left(Q_{m+1}, \ldots, Q_{n}\right)$ are the generalized force vectors dual to $v^{\circ}$ and $v^{*}$ respectively. On the other hand, the input-output relation between $Q^{\circ}$ and $Q^{*}$ is reverse to $v^{\circ}$ and $v^{*}$; namely, $Q^{\circ}$ is the input and $Q^{*}$ the output. In the above, the orthogonality condition holds:

$B^{T}(q) N^{T}(q)=0$.

The matrices $N(q)$ and $B(q)$ are called connection matrices (see Yoshimura, 1995). This orthogonality condition denotes principle of virtual work, which is given by

$\langle Q, v\rangle=0$, for all $v \in \Delta(q)$ and $Q \in \Delta^{\circ}(q)$,

where $\langle$,$\rangle denotes a duality pairing.$

The dual set of constraints given by Eqs. (4) and (5) indicates structural relations, namely, it represents how physical elements are interconnected. Thus, we sometimes call the structural relation an interconnection among the physical elements. In circuit theory, it is known that Eqs. (4) and (5) correspond to KCL and KVL and also that the virtual work principle is known as Tellegen's theorem. Furthermore, there exists a relation called duality principle as in Fig. 1, which is known as Planck-Okada-Arsove principle in circuit theory (see Yoshimura, 1995).

\subsection{Implicit DAEs for Lagrangian systems}

Here, we show how the notion of Dirac structures can be fit into the formulation of implicit Lagrangian systems (see Yoshimura and Marsden, 2006a,b, 2008). Let $L$ be a Lagrangian on $T Q$, which is given by

$L(q, \dot{q})=\frac{1}{2}\left\langle\dot{q}^{\circ}, M \dot{q}^{\circ}\right\rangle-U\left(q^{\circ}\right)$,

where we assume that $L$ is only associated to $\dot{q}^{\circ}, M$ is a mass matrix whose components are functions of $q^{\circ}$, and $U$ denotes a potential energy function of $q^{\circ}$. This implies that $L$ is possibly degenerate. 
The Lagrange-d'Alembert principle is given by

$\int_{a}^{b}\left\langle\frac{\mathrm{d}}{\mathrm{d} t} \frac{\partial L}{\partial \dot{q}}-\frac{\partial L}{\partial q}, \delta q\right\rangle \mathrm{d} t+\int_{a}^{b}\langle F, \delta q\rangle \mathrm{d} t=0$,

where $\delta q$ satisfies the constraint

$N(q) \delta q=o_{m}$.

So, one can obtain the dual dynamical relation

$B^{T}(q) Q=o_{n-m}$,

where

$Q=\frac{\mathrm{d}}{\mathrm{d} t} \frac{\partial L}{\partial \dot{q}}-\frac{\partial L}{\partial q}+F$

and it directly induces

$Q=\left[\begin{array}{l}Q^{\circ} \\ Q^{*}\end{array}\right]=\left[\begin{array}{l}M \dot{v}^{\circ}+f\left(q^{\circ}, v^{\circ}\right)-\frac{\partial U}{\partial q^{\circ}} \\ \tau\end{array}\right]$,

where

$\frac{\mathrm{d}}{\mathrm{d} t} \frac{\partial L}{\partial \dot{q}}-\frac{\partial L}{\partial q}=\left[\begin{array}{l}M \dot{v}^{\circ}+f\left(q^{\circ}, v^{\circ}\right)-\frac{\partial U}{\partial q^{\circ}} \\ 0\end{array}\right]$

and

$F=\left[\begin{array}{l}0 \\ \tau\end{array}\right]$.

Notice that $\tau$ indicates the external forces. Of course, equations of motion can be written as

$B^{T}(q)\left(\frac{\mathrm{d}}{\mathrm{d} t} \frac{\partial L}{\partial \dot{q}}-\frac{\partial L}{\partial q}+F\right)=o_{n-m}$.

Furthermore, one has the second-order condition (see Marsden and Ratiu, 1999):

$\dot{q}^{\circ}-v^{\circ}=o_{m}$,

$\dot{q}^{*}-v^{*}=o_{n-m}$.

From Eqs. (4), (5), (6), (7) and (8), we can obtain the following local differential-algebraic equations

$G(x(t), \dot{x}(t) ; u(t))=0$,

where $x=(q, v, Q)=\left(q^{\circ}, q^{*}, v^{\circ}, v^{*}, Q^{\circ}, Q^{*}\right) \in W \times W \times W^{*}$ denotes the state variables and $u=\tau \in\left(W^{n-m}\right)^{*}=\left(\mathbb{R}^{n-m}\right)^{*}$ the input variables. In the above, we locally set $T Q \cong W \times W$ and $T^{*} Q \cong W \times W^{*}$, and hence $T Q \oplus T^{*} Q \cong W \times W \times W^{*}$, where $Q \cong W=W^{m} \times W^{n-m}=\mathbb{R}^{m} \times \mathbb{R}^{n-m}$ is an $n$-dimensional vector space which is a model space for $Q$. Thus, the mathematical model of the Lagrangian system is given by the implicit DAEs:

$G=\left[\begin{array}{l}G_{1} \\ G_{2} \\ G_{3} \\ G_{4} \\ G_{5} \\ G_{6}\end{array}\right]=\left[\begin{array}{l}\dot{q}^{\circ}-v^{\circ} \\ \dot{q}^{*}-v^{*} \\ v^{\circ}-\mathcal{J} v^{*} \\ \mathcal{J}^{T} Q^{\circ}+Q^{*} \\ Q^{\circ}-M \dot{v}^{\circ}-f\left(q^{\circ}, v^{\circ}\right)-\partial U / \partial q^{\circ} \\ Q^{*}-\tau\end{array}\right]$.

\section{Sparse tableau approach}

From the viewpoint of numerical analysis for mechanical systems, there exist two kinds of dynamical problems; namely, the forward dynamics and inverse dynamics.

Recall that $W=W^{m} \times W^{n-m}=\mathbb{R}^{m} \times \mathbb{R}^{n-m}$ is the model space for $Q$. The forward dynamics analysis is the case in which given a smooth input vector

$u(t):=\tau(t) \in\left(W^{n-m}\right)^{*}=\left(\mathbb{R}^{n-m}\right)^{*}$

as a vector function of time $t$, numerically integrate Eq. (9) in terms of $t$ to obtain

$x(t)=\left(q^{\circ}(t), q^{*}(t), v^{\circ}(t), v^{*}(t), Q^{\circ}(t), Q^{*}(t)\right)$

as the output, where $x \in W \times W \times W^{*}$. On the other hand, the inverse dynamics analysis is the case in which given a smooth input vector

$u(t):=q^{*}(t) \in W^{n-m}=\mathbb{R}^{n-m}$

as a vector function of time $t$, then compute

$x(t)=\left(q^{\circ}(t), v^{\circ}(t), v^{*}(t), Q^{\circ}(t), Q^{*}(t), \tau(t)\right)$

as the output, in which case $x \in W^{m} \times W \times W^{*} \times\left(W^{n-m}\right)^{*}$.

In this paper, we explore the case of the inverse dynamics analysis by the sparse tableau approach, where the state vector is given by $x=\left(q^{\circ}, v^{\circ}, v^{*}, Q^{\circ}, Q^{*}, \tau\right)$. To do this, let us first discretize Eq. (9) at time $t=t_{n}$. By using the Backward Differentiation Formula $(B D F)$ developed by Gear (see, for instance, Brennen et al., 1995), the time-derivative term $\dot{x}_{n}=\dot{x}\left(t_{n}\right)$ associated to the state vector $x_{n}=x\left(t_{n}\right)$ may be approximately discretized by the backwards $x_{n-i}=x\left(t_{n-i}\right)$ as

$\dot{x}_{n}=-\sum_{i=0}^{k} \frac{1}{h} \alpha_{i} x_{n-i} \quad(1 \leq k \leq 6)$,

where $h=t_{n}-t_{n-1}$ denotes a time step, $k$ is a backward order and $\alpha_{i}$ indicates the coefficient associated to the $i$-th backward order. Substituting Eq. (11) into Eq. (9), we obtain the discretized nonlinear algebraic equations as follows:

$G\left(x_{n}, \dot{x}\left(x_{n}\right) ; u\left(t_{n}\right)\right)=0$.

Recall the algorithm of the Sparse Tableau Approach is given in Fig. 2 (see Hachtel et al., 1971; Brayton et al., 1972), where we linearize Eq. (12) at each time step $t=t_{n}$ as

$J\left(x_{n}^{(r)}\right) \triangle x_{n}^{(r)}=-G\left(x_{n}^{(r)}\right)$,

and where $J=\left.\left[\partial G_{i} / \partial x_{j}\right]\right|_{t=t_{n}}$ is the Jacobian matrix and $\Delta x_{n}^{(r)}$ denotes the $r$-th iterated corrector vector at $t_{n}$. Then, it follows

$$
\begin{aligned}
x_{n}^{(r+1)} & =x_{n}^{(r)}+\Delta x_{n}^{(r)} \\
& =x_{n}^{(r)}-J^{-1}\left(x_{n}^{(r)}\right) G\left(x_{n}^{(r)}\right) .
\end{aligned}
$$




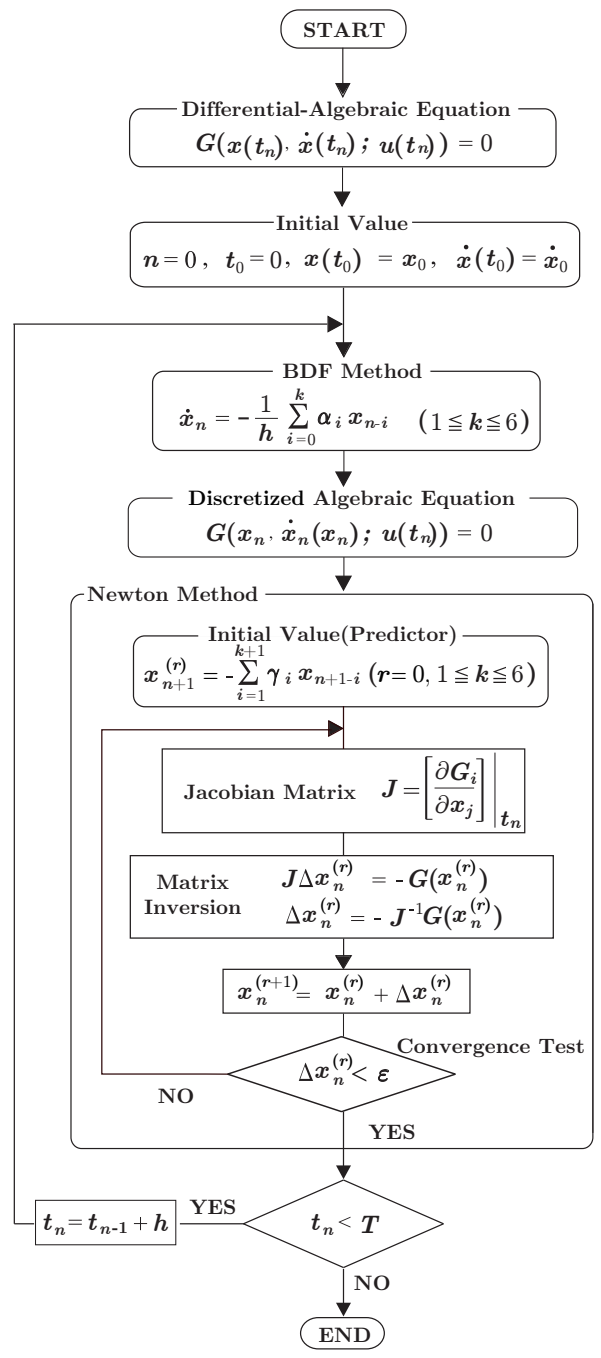

Figure 2. Sparse tableau method.

In the Newton method, it is necessary to take initial values near from the solution $x_{n}$ and the $k$-th prediction formula $x_{n}^{P r}$ is given by

$x_{n}^{P r}=x_{n}^{(0)}=-\sum_{i=1}^{k} \gamma_{i} x_{n-i}$,

where $\gamma_{i}$ is the $i$-th coefficient.

In the inverse dynamics analysis, the state vector is given by $x=\left(q^{\circ}, v^{\circ}, v^{*}, Q^{\circ}, Q^{*}, \tau\right)$ and it follows from Eq. (10) that the Jacobian matrix is given as in Fig. 3, where $I_{n}$ stands for the $n$-th degree unit matrix.

\section{Bipartite graphs}

\subsection{Input-output relations}

The Jacobian matrix $J\left(x_{n}^{(r)}\right)$ obtained in Eq. (13) apparently has the characteristic of random sparseness. So, we develop

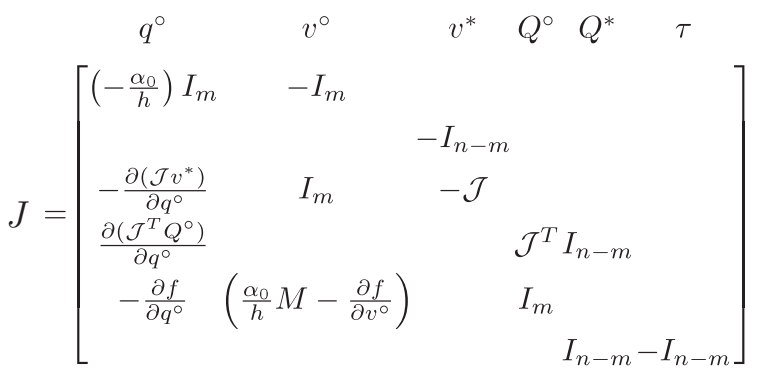

Figure 3. Jacobian matrix.

an efficient symbolic generation for computations of the sparse Jacobian matrix inversion for Newton's iterations. To do this, let us consider an input-output relation among state variables for every relation in (10). Now, we can uniquely allocate the input-output relation to the kinematical and dynamical relations in Eqs. (4) and (5) as follows:

$v^{\circ}-\mathcal{J} v^{*}=o_{m} \quad$ (output $: v^{\circ}$, input : $\left.v^{*}\right)$,

$\mathcal{J}^{T} Q^{\circ}+Q^{*}=o_{n-m}$ (input : $Q^{\circ}$, output : $Q^{*}$ ).

Similarly, for the second-order conditions between $v=$ $\left(v^{\circ}, v^{*}\right)$ and $\dot{q}=\left(\dot{q}^{\circ}, \dot{q}^{*}\right)$, one has

$$
\begin{array}{ll}
\dot{q}^{\circ}-v^{\circ}=o_{m} & \text { (output : } \left.\dot{q}^{\circ}, \text { input }: v^{\circ}\right), \\
\dot{q}^{*}-v^{*}=o_{n-m} & \text { (input } \left.: \dot{q}^{*}, \text { output }: v^{*}\right) .
\end{array}
$$

Furthermore, as to the equations of motion, it follows

$$
\begin{array}{ll}
Q^{\circ}-M \dot{v}^{\circ}-f\left(q^{\circ}, v^{\circ}\right) \\
\quad-\partial_{q^{\circ}} U=o_{m} & \text { (output: } \left.Q^{\circ}, \text { input : } q^{\circ}, v^{\circ}\right), \\
Q^{*}-\tau=o_{n-m} & \text { (input : } \left.Q^{*}, \text { output : } \tau\right) .
\end{array}
$$

The input-output relations in the mentioned above can be determined by physical causality. Needless to say that the time derivative terms $\dot{x}\left(t_{n}\right)$ are expressed in terms of the backwards $x_{n-i}\left(t_{n}\right), i=0, \ldots, 6$ by using the BDF as in equation (11). Corresponding to the Jacobian matrix $J$ in (13), define the causal Jacobian matrix $\hat{J}$ by assigning -1 to the input and +1 to the output as to the $j$-th variable in the $i$-th relation associated to $J_{i j}$ as

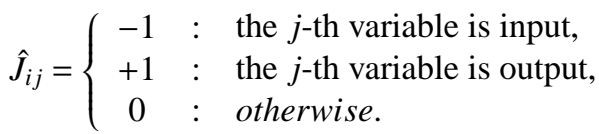

Thus, the causal Jacobian matrix $\hat{J}$ is given in Fig. 4 .

In the above, $\widehat{I}_{m}$ indicates the $m$-th unit matrix. Note that there exists an element with +1 in each row, which plays a role of the pivot in the Gaussian elimination. Further, $\widehat{I}_{m, n-m}$ is the $m \times(n-m)$ matrix, in which +1 are allocated to non-zero components of the $m \times(n-m)$ matrix $\mathcal{J}$.

\subsection{Oriented bipartite graphs}

Let us illustrate the input-output relations as to the Jacobian matrix $J=\left[J_{i j}(x)\right]=\left.\left[\partial G_{i} / \partial x_{j}\right]\right|_{t=t_{n}}$ by introducing 


$$
\widehat{J}=\left[\begin{array}{cccccc}
q^{\circ} & v^{\circ} & v^{*} & Q^{\circ} & Q^{*} & \tau \\
+I_{m} & -I_{m} & & & \\
\multicolumn{5}{c}{+I_{n-m}} \\
-\widehat{I}_{m} & +I_{m}-\widehat{I}_{m, n-m} & & \\
-\widehat{I}_{n-m, m} & & \multicolumn{3}{c}{-\widehat{I}_{n-m, m}+I_{n-m}} \\
-\widehat{I}_{m} & -\widehat{I}_{m} & & & +I_{m} & \\
& & & & & -I_{n-m}+I_{n-m}
\end{array}\right]
$$

Figure 4. Causal Jacobian matrix.

bipartite graphs. Recall that the $i$-th row of the Jacobian matrix $J=\left[J_{i j}(x)\right]=\left.\left[\partial G_{i} / \partial x_{j}\right]\right|_{t=t_{n}}$ corresponds to the $i$-th vector function $G_{i}(x, \dot{x} ; u)$ and the $j$-th column corresponds to $j$-th vector $x_{j}$ respectively, where $i, j=1, \ldots, \mathcal{N}(=3 n)$. Here, $G_{i}(x, \dot{x} ; u)=0$ are the $\mathcal{N}$-th order DAEs and $x=\left(x_{1}, \ldots, x_{6}\right)=$ $\left(q^{\circ}, v^{\circ}, v^{*}, Q^{\circ}, Q^{*}, \tau\right)$ is the $\mathcal{N}$-vector.

Given the causal Jacobian matrix $\hat{J}$ associated to $J$, let $V_{r}=\left\{G_{1}, G_{2}, \ldots, G_{\mathcal{N}}\right\}$ be the row-set of $\hat{J}$ and let $V_{c}=$ $\left\{\Delta x_{1}, \ldots, \Delta x_{6}\right\}$ be the column-set of $\hat{J}$. Here, we assign $V_{r}$ and $V_{c}$ to vertex set $V:=V_{r} \times V_{c}$.

Now, there are nonzero elements $( \pm 1)$ in the $i$-th row of the causal Jacobian matrix and suppose that they are in the $k$-th and $l$-th columns. For instance, as to the first equation $G_{1}=0$, one has the kinematical relation between $x_{1}=q^{\circ}$ and $x_{2}=v^{\circ}$ as $\dot{x}_{1}-x_{2}=0$. By the BDF discretization, the corresponding linearized equation is given as

$-\frac{1}{h}\left(\alpha_{0} \Delta x_{1}\right)-\Delta x_{2}=0$.

In order to illustrate input-output relations among the state variables by graphs, let us define arc-set by

$A=\left\{(j, i) \mid \hat{J}_{i j} \neq 0, i \in V_{c}, j \in V_{r}\right\}$,

where the arcs represent some relations between vertices in $V_{c}$ (state variables) as to every vertex (equation) in $V_{r}$. Furthermore, the direction of an arc indicates causality or an input-output relation among state variables associated to the column vertices. Let $a \in A$ be an arc. Let us introduce a map $s: A \rightarrow V$, which is given by, for an arc $a \in A, s(a)$ denotes the initial vertex of $a$. Similarly, define a map $t: A \rightarrow V$, which is given by, for an arc $a \in A, t(a)$ indicates the terminal vertex of $a$. So, we can define the set of vertices incident to $a$ by $\{s(a), t(a)\}$. Sometimes, $s(a)$ is called the source of $a$ and $t(a)$ the target of $a$. Hence, we can define a directed bipartite graph by

$B=\left(V_{r}, V_{c}, A, s, t\right)$,

by which we can illustrate the input-output relations associated to the Jacobian matrix as shown in Fig. 5.

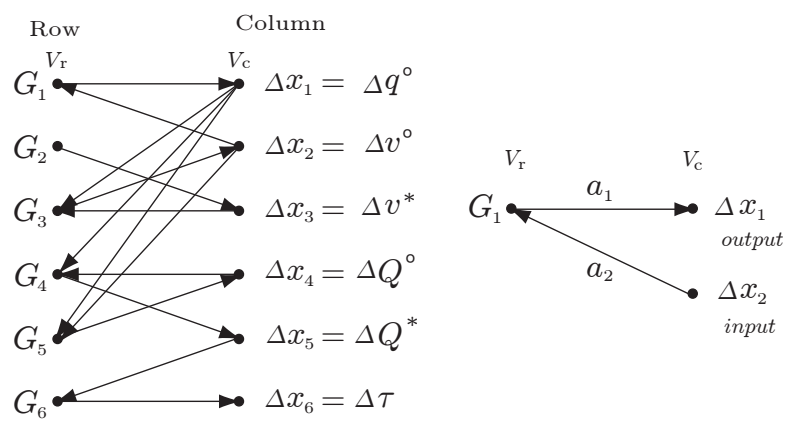

Figure 5. Directed bipartite graph.

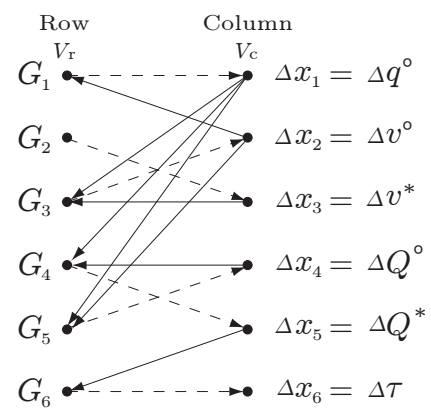

Figure 6. Perfect matching.

\subsection{Perfect matching}

A matching $M \subset A$ of a bipartite graph $B=\left(V_{r}, V_{c}, A, s, t\right)$ is defined by a set of arcs without common vertices. Especially, $M$ is called a perfect matching if it is a matching which matches all vertices of the graph, namely, every vertex of the graph is incident to exactly one edge of the matching: $s(M)=$ $V_{r}$ and $t(M)=V_{c}$.

In Fig. 6, the arcs that are drawn by the broken lines consist of the perfect matching. If a perfect matching exists in $B$, the corresponding Jacobian matrix $J$ is a square and nonsingular matrix since $\left|V_{r}\right|=\left|V_{c}\right|$. Here, $|V|$ means the size of $V$, namely, the number of elements in the set of vertices $V$.

Let us see that $\left|V^{+}\right|=\left|V^{-}\right|$is equivalent with the fact that the Jacobian matrix is diagonalizable. First, let us show the following relation:

Perfect matchings exist in $B \Rightarrow J$ is diagonalizable.

This is clear because a perfect matching can be detected by the elementary row and column operations in matrix; namely, (1) interchanging two rows or columns; (2) adding a multiple of one row or column to another; (3) multiplying any row or column by a nonzero element, although there might be several perfect matchings for a given bipartite graph. Next, let us show the following relation:

\section{$J$ is diagonalizable $\Rightarrow$ Perfect matchings exist in $B$.}

That the Jacobian matrix $J$ is diagonalizable means that one can properly select a pivot in each row or column from 
nonzero elements of the matrix. In other words, the rank of $J$ is equal to $\mathcal{N}$. Recall that a cover is defined as a pair $\left(\bar{V}_{r}, \bar{V}_{c}\right)$ of $\bar{V}_{r} \subset V_{r}$ and $\bar{V}_{c} \subset V_{c}$ such that there exist no arcs between $V_{r} \backslash \bar{V}_{r}$ and $V_{c} \backslash \bar{V}_{c}$. For the case in which Rank $J=$ $\mathcal{N}=|V| / 2$, the number of the minimum cover for the vertex set $V=\left(V_{r}, V_{c}\right)$ of the bipartite graph $B$ is to be $\mathcal{N}$. It follows from the König-Egerváry theorem that the number of arcs in a maximum matching is equal to the number of vertices in a minimum vertex cover; namely,

$$
\begin{aligned}
\max \{|M| \mid M: & \text { matching }\} \\
& =\min \left\{\left|\bar{V}_{r}\right|+\left|\bar{V}_{c}\right| \mid\left(\bar{V}_{r}, \bar{V}_{c}\right): \text { cover }\right\} .
\end{aligned}
$$

So, the size of the maximum matching is to be $\mathcal{N}=|V| / 2$ and therefore we have shown that the matching is a perfect matching. As shown in Fig. 6, there exists a perfect matching

$$
\begin{aligned}
M=\left\{a_{1}=\left(x_{1}, G_{1}\right), a_{2}=\left(x_{2}, G_{3}\right), a_{3}=\left(x_{3}, G_{2}\right),\right. \\
\\
\left.a_{4}=\left(x_{4}, G_{5}\right), a_{5}=\left(x_{5}, G_{4}\right), a_{6}=\left(x_{6}, G_{5}\right)\right\} \subset A .
\end{aligned}
$$

For each arc $a \in M$, one can choose the target $x=t(a)$ as the pivot associated to the $k$-th equation, where $k=s(a)$ is the source of $a$. Thus, the proof has been done.

Therefore, we can conclude the following relation:

$$
\text { Perfect matchings exist in } B \Leftrightarrow J \text { is diagonalizable. }
$$

In this way, the solvability of the linearized Eq. (13) is clarified in the context of the perfect matching by using the bipartite graph associated to the causal Jacobian matrix. Recall that nonzero elements of $\hat{J}$ correspond to arcs of the bipartite graph and hence the number of nonzero elements and the number of the arcs are the same. In our study, in each row, there is only one output variable, which implies that the arcs associated with the output variables never share the vertices of other output arcs in the bipartite graph. Therefore, the set of edges of output variables has perfect matching.

\section{Sparse matrix inversion}

\subsection{Symbolic generation}

Next, we show how the sparse matrix inversion of the Jacobian matrix can be done by symbolic manipulation. By the information from the causal Jacobian matrix, selecting the pivot, the Jacobian matrix can be diagonalized as shown in Fig. 7, where $G^{(k)}$ is the $k$-th tableau error vector, $A^{(k)}$ represents a matrix in the Jacobian matrix for the $k$-th step of elementary operations. Next, after forward Gaussian elimination for the Jacobian matrix, we can easily obtain the reduced Jacobian matrix as in Fig. 8. Note that we can do this by symbolic manipulation. Furthermore, after backward elimination as to the reduced Jacobian matrix by symbolic manipulation,

$$
\left[\begin{array}{ccccc}
-I_{n-m} & & & & \\
A_{1}^{(1)} & I_{m} & A_{2}^{(1)} & & \\
& -I_{m} & A_{3}^{(1)} & & \\
& A_{4}^{(1)} & A_{5}^{(1)} & I_{m} & \\
& & A_{6}^{(1)} & A_{7}^{(1)} & I_{n-m} \\
& & & & I_{n-m}-I_{n-m}
\end{array}\right]\left[\begin{array}{c}
\Delta v^{*} \\
\Delta v^{\circ} \\
\Delta q^{\circ} \\
\Delta Q^{\circ} \\
\Delta Q^{*} \\
\Delta \tau
\end{array}\right]=-\left[\begin{array}{c}
G_{1}^{(1)} \\
G_{2}^{(1)} \\
G_{3}^{(1)} \\
G_{4}^{(1)} \\
G_{5}^{(1)} \\
G_{6}^{(1)}
\end{array}\right]
$$

Figure 7. Diagonalized Jacobian matrix.

$$
\left[\begin{array}{ccccc}
-I_{n-m} & & & & \\
& & & & \\
& I_{m} A_{2}^{(1)} & & & \\
& A_{3}^{(2)} & & & \\
& & I_{m} & & \\
& & & I_{n-m} & \\
& & & & -I_{n-m}
\end{array}\right]\left[\begin{array}{c}
\Delta v^{*} \\
\Delta v^{\circ} \\
\Delta q^{\circ} \\
\Delta Q^{\circ} \\
\Delta Q^{*} \\
\Delta \tau
\end{array}\right]=-\left[\begin{array}{c}
G_{1}^{(1)} \\
G_{2}^{(2)} \\
G_{3}^{(2)} \\
G_{4}^{(3)} \\
G_{5}^{(3)} \\
G_{6}^{(2)}
\end{array}\right]
$$

Figure 8. Reduced Jacobian matrix.

we can obtain the following corrector vector consequently:

$$
\begin{aligned}
& \Delta \tau=G_{6}^{(2)}, \\
& \Delta Q^{*}=-G_{5}^{(3)}, \\
& \Delta Q^{\circ}=-G_{4}^{(3)}, \\
& \Delta q^{\circ}=-\frac{A_{2}^{(2)}}{G_{3}^{(2)}}, \\
& \Delta v^{\circ}=-\left(G_{2}^{(2)}-A_{2}^{(1)} \cdot G_{3}^{(2)}\right), \\
& \Delta v^{*}=G_{1}^{(1)} .
\end{aligned}
$$

Thus, we can develop the inversion of the Jacobian matrix, i.e., $\Delta x_{n}^{(r)}=-J^{-1}\left(x_{n}^{(r)}\right) G\left(x_{n}^{(r)}\right)$ can be explicitly done by symbolic manipulation with the causal information of $\hat{J}$. As a result, we can make symbolic generation of Eq. (14).

\subsection{Numerical analysis}

Let us demonstrate the validly of our symbolic generation scheme by an example of the Stanford manipulator with 6 degrees of freedom as shown in Fig. 9. One can set up 96, $150,210,390,738$ system equations of DAE models for the stanford manipulator, where the numbers of systems equations correspond to those of the unknown variables according to the elimination of redundant variables.

We examine to compare the CPU time required in the routine of the Jacobian matrix inversion in each time step for the three cases: (1) Ordinary Gaussian elimination method (without any sparse matrix algorithm), (2) Sparse Gaussian elimination method of the inner product algorithm (as to the opensource subroutine, see Murata et al., 1985), (3) our method. The comparison of the required CPU time is illustrated in Fig. 10. As a result, our method of sparse matrix inversions 


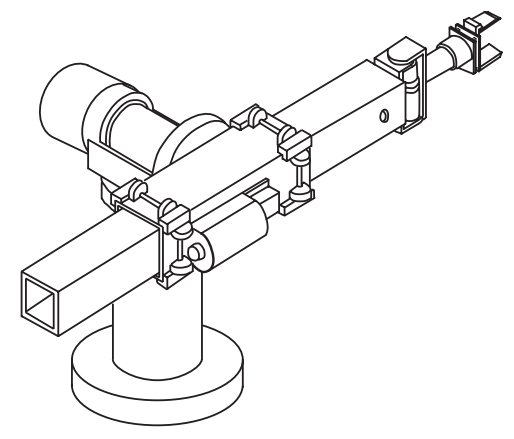

Figure 9. Stanford manipulator.

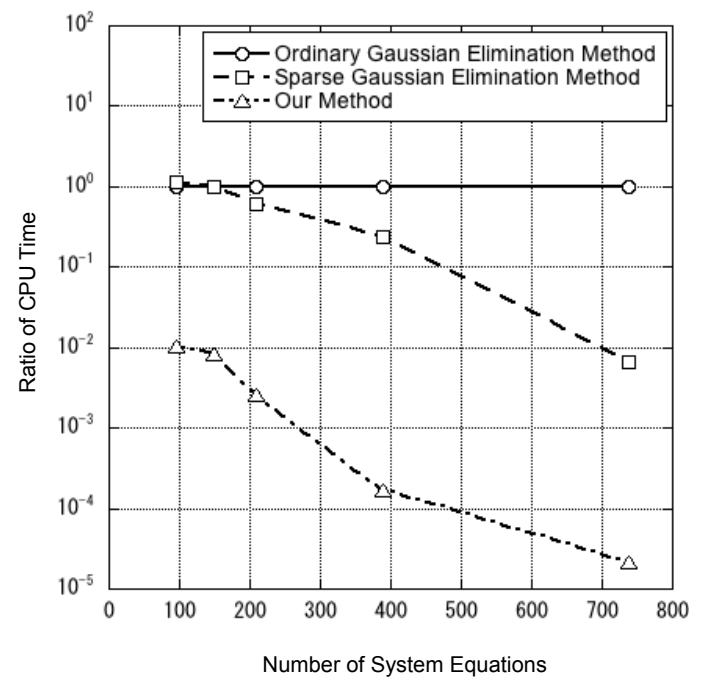

Figure 10. Comparison of CPU time.

has a great advantage in the CPU time efficiency in comparison with other approaches.

\section{Conclusions}

We have shown symbolic generation of sparse matrix inversion for large-scale mechanical systems. We have set up Implicit DAE models in the context of Lagrangian systems and we have shown the input-output relations as to the Jacobian matrix associated with linearized equations. Then, we have shown the solvability of the linearized equations by using the bipartite graph. Furthermore, we have proposed symbolic generation of the random sparse matrix inversion for the Jacobian matrix. Finally, we have demonstrated the validity of our approach by numerical analysis with an example of the Stanford manipulator comparing with the inner-product sparse matrix Gaussian elimination algorithm as well as the standard Gaussian elimination algorithm.
Acknowledgements. This research is partially supported by JSPS Grant-in-Aid 23560269, JST-CREST, Waseda University Grant for SR 2012A-602 and IRSES project Geomech-246981.

Edited by: A. Müller

Reviewed by: two anonymous referees

\section{References}

Brayton, R. K., Gustavson, F. G., and Hachtel, G. D.: A new efficient algorithm for solving differential-algebraic systems using implicit backward differentiation formulas, Proc. IEEE, 60, 98108, 1972.

Brennen, K. E., Campbell, S. L., and Petzold, L. R.: Numerical Solution of Initial-Value Problems in Differential-Algebraic Equations, Philadelphia, SIAM, 1995.

Hachtel, G. D., Brayton, R. K., and Gustavson, F. G.: The sparse tableau approach to network analysis and design, IEEE Trans. Circuit Theory, CT-18, 101-113, 1971.

Marsden, J. E. and Ratiu, T. S.: Introduction to Mechanics and Symmetry, volume 17 of Texts in Applied Mathematics, SpringerVerlag, 2nd Edn., 1999.

Murata, T., Oguni, T., and Karaki, Y.: Supercomputer-Application to Scientific Computing, Maruzen, 1985 (in Japanese).

Murota, K.: Matrices and Matroids for Systems Analysis, SpringerVerlag, Berlin, 2000.

Orlandea, N., Chace, M. A., and Calahan, D. A.: A sparsity-oriented approach to the dynamic analysis and design of mechanical systems - Part 1, Part 2, Trans. ASME, J. Eng. Ind., 99, 773-779, 780-784, 1977.

Yoshimura, H.: Dynamics of Flexible Multibody Systems, Ph.D. thesis, Waseda University, Tokyo, Japan, 1995.

Yoshimura, H. and Imai, N.: A sparse tableau approach to dynamics of large-scale nonlinear mechanical systems, IEICE Nonlinear Problems, 103, 39-43, 2004.

Yoshimura, H. and Marsden, J. E.: Dirac structures in Lagrangian mechanics Part I: Implicit Lagrangian systems, J. Geom. Phys., 57, 133-156, 2006a.

Yoshimura, H. and Marsden, J. E.: Dirac structures in Lagrangian mechanics Part II: Variational structures, J. Geom. Phys., 57, 209-250, 2006b.

Yoshimura, H. and Marsden, J. E.: Representations of Dirac Structures and Implicit Port-controlled Lagrangian Systems, Proc. International Symposium on Mathematical Theory of Networks and Systems 2008, Blacksburg, Virginia, 1-12, 2008. 